262.

\title{
ON THE EQUATION OF DIFFERENCES FOR AN EQUATION OF ANY ORDER, AND IN PARTICULAR FOR THE EQUATIONS OF THE ORDERS TWO, THREE, FOUR, AND FIVE.
}

[From the Philosophical Transactions of the Royal Society of London, vol. CL. (for the year 1860), pp. 93-112. Received March 2,-Read March 29, 1860.]

THE term, equation of differences, denotes the equation for the squared differences of the roots of a given equation; the equation of differences afforded a means of determining the number of real roots, and also limits for the real roots, of a given numerical equation, and was upon this account long ago sought for by geometers. In the Philosophical Transactions for 1763, Waring gives, but without demonstration or indication of the mode of obtaining it, the equation of differences for an equation of the fifth order wanting the second term: the result was probably obtained by the method of symmetric functions. This method is employed in the Meditationes Algebraicce (1782), where the equation of differences is given for the equations of the third and fourth orders wanting the second terms; and in p. 85 the before-mentioned result for the equation of the fifth order wanting the second term, is reproduced. The formulæ for obtaining by this method the equation of differences, are fully developed by Lagrange in the Traité des Equations Numériques (1808); and he finds by means of them the equation of differences for the equations of the orders two and three, and for the equation of the fourth order wanting the second term; and in Note III. he gives, after Waring, the result for the equation of the fifth order wanting the second term. It occurred to me that the equation of differences could be most easily calculated by the following method. The coefficients of the equation of differences, qud functions of the differences of the roots of the given equation, are leading 
coefficients of covariants, or (to use a shorter expression) they are "Seminvariants( ${ }^{1}$ )," that is, each of them is a function of the coefficients which is reduced to zero by one of the two operators which reduce an invariant to zero. In virtue of this property they can be calculated, when their values are known for the particular case in which one of the coefficients of the given equation is zero. To fix the ideas, let the given equation be $(* \gamma v, 1)^{n}=0$; then, when the last coefficient or constant term vanishes, the equation breaks up into $v=0$ and into an equation of the degree $n-1$, which I call the reduced equation; the equation of differences will break up into two equations, one of which is the equation of differences for the reduced equation, the other is the equation for the squares of the roots of the same reduced equation. This hardly requires a proof; let the roots of the given equation be $\alpha, \beta, \gamma, \delta$, \&c., those of the equation of differences are $(\alpha-\beta)^{2},(\alpha-\gamma)^{2},(\alpha-\delta)^{2}$, \&c., $(\beta-\gamma)^{2},(\beta-\delta)^{2},(\gamma-\delta)^{2}$, \&c. ; but in putting the constant term equal to zero, we in effect put one of the roots, say $\alpha$, equal to zero; the roots of the equation of differences thus become $\beta^{2}, \gamma^{2}, \delta^{2}$ \&c., $(\beta-\gamma)^{2},(\beta-\delta)^{2},(\gamma-\delta)^{2}$, \&c. The equation for the squares of the roots can be found without the slightest difficulty; hence if the equation of differences for the reduced equation of the order $n-1$ is known, we can, by combining it with the equation for the squares of the roots, form the equation of differences for the given equation with the constant term put equal to zero, and thence by the above-mentioned property of the Seminvariancy of the coefficients, find the equation of differences for the given equation. The present memoir shows the application of the process to equations of the orders two, three, four, and five: part of the calculation for the equation of the fifth order was kindly performed for me by the Rev. R. Harley. It is to be noticed that the best course is to apply the method in the first instance to the forms $(a, b, \ldots\} v, 1)^{n}=0$, without numerical coefficients (or, as they may be termed, the denumerate forms), and to pass from the results so obtained to those which belong to the forms $\left(a, b, \ldots \gamma(v, 1)^{n}=0\right.$, or standard forms. The equation of differences, for $(\alpha-\beta)^{2}$, \&c., the coefficients of which are seminvariants, naturally leads to the consideration of a more general equation having for its roots $(\alpha-\beta)^{2}(x-\gamma y)^{2}(x-\delta y)^{2} \ldots$, \&c., the coefficients of which are covariants; and in fact, when, as for equations of the orders two, three, and four, all the covariants are known, such covariant equation can be at once formed from the equation of differences; for equations of the fifth order, however, where the covariants are not calculated beyond a certain degree, [they are now all calculated, see 141 and 143], only a few of the coefficients of the covariant equation can be thus at once formed. At the conclusion of the memoir, I show how the equation of differences for an equation of the order $n$ can be obtained by the elimination of a single quantity from two equations each of the order $n-1$; and by applying to these two equations the simplification which I have made in Bezout's abridged method of elimination, I exhibit the equation of differences for the given equation of the order $n$, in a compendious form by means of a determinant; the first-mentioned method is, however, that which is best adapted for the actual development of the equation of differences for the equation of a given order.

1 The term "Seminvariant" seems to me preferable to M. Brioschi's term "Peninvariant."

C. IV. 
The equations successively considered are

$$
\begin{array}{lll}
(a, b, c & \gamma v, 1)^{2}=0, \\
(a, b, c, d & \gamma v, 1)^{3}=0, \\
(a, b, c, d, e & \gamma v, 1)^{4}=0, \\
\left(a, b, c, d, e, f^{\gamma} v, 1\right)^{5}=0 .
\end{array}
$$

The equation of differences for the quadric, and that for the squares of the roots, are considered to be known, and the cther results are derived from them: it will be convenient to write down in the first instance the results for the quadric, the cubic, and the quartic equations, and then explain the process of obtaining them.

For the quadric equation,

Equation of differences is, $0=$

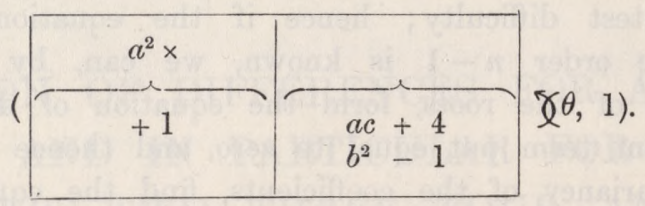

Equation for the squares of the roots is, $0=$

\begin{tabular}{|c|c|c|c|}
\hline$a^{4} \times$ & $a^{2} \times$ & & \\
\hline+1 & $\begin{array}{l}a c+6 \\
b^{2}-2\end{array}$ & $\begin{array}{l}a^{2} c^{2}+9 \\
a b^{2} c-6 \\
b^{4}+1\end{array}$ & $\begin{array}{l}a^{2} d^{2}+27 \\
a b c d-18 \\
a c^{3}+4 \\
b^{3} d+4 \\
b^{2} c^{2}-1\end{array}$ \\
\hline
\end{tabular}

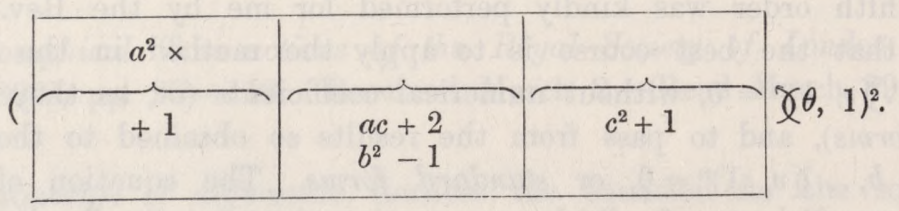

For the cubic equation,

Equation of differences is, $0=$

Equation for the squares of the roots is, $0=$

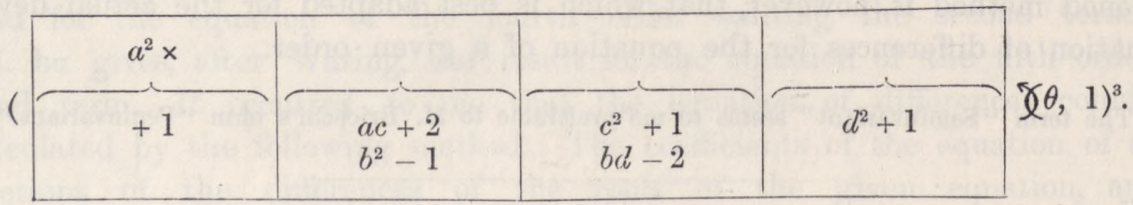


For the quartic equation,

Equation of differences is, $0=$

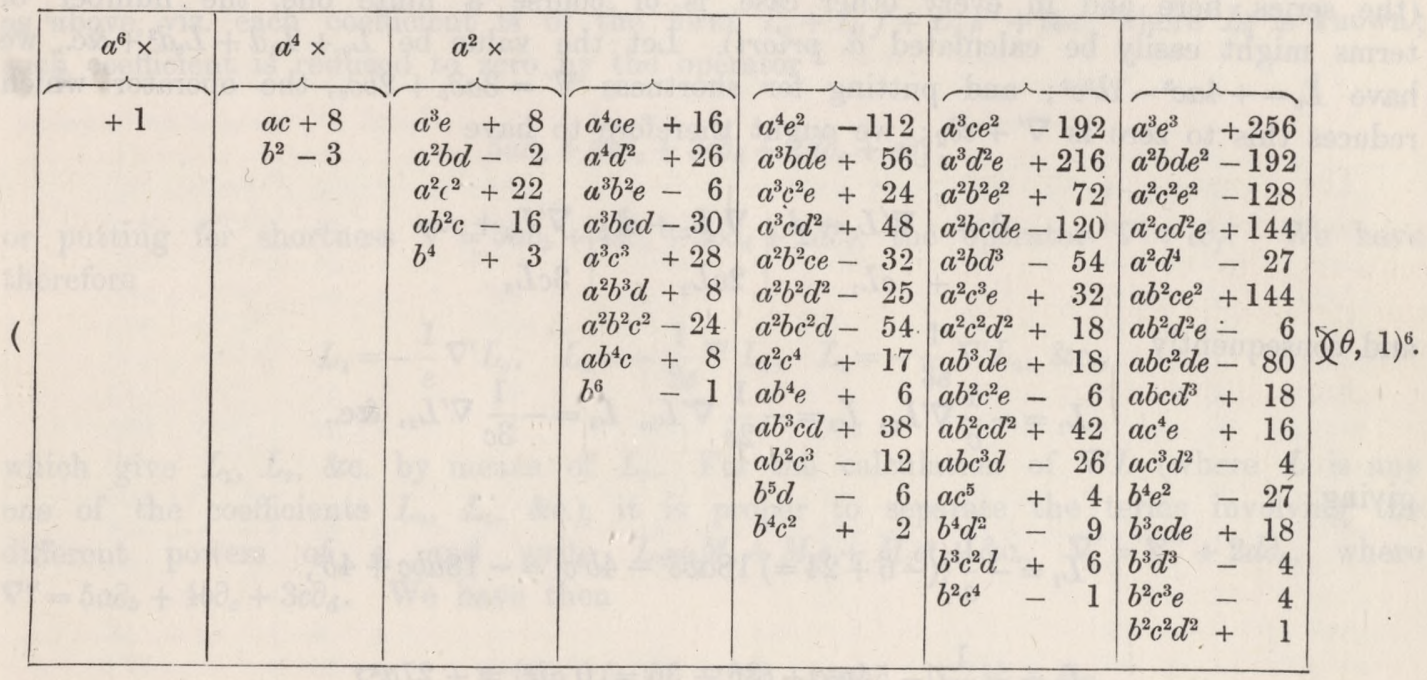

Equation for squares of the roots is, $0=$

\begin{tabular}{|c|c|c|c|c|}
\hline$a^{2} \times$ & & & & \\
\hline+1 & $\begin{array}{l}a c+2 \\
b^{2}-1\end{array}$ & $\begin{array}{l}a e+2 \\
b d-2 \\
c^{2}+1\end{array}$ & $\begin{array}{l}c e+2 \\
d^{2}-1\end{array}$ & $e^{2}+1$ \\
\hline
\end{tabular}

The multiplication of the equation of differences and the equation of the squares of the roots of the quadric equation, gives the equation, $0=$

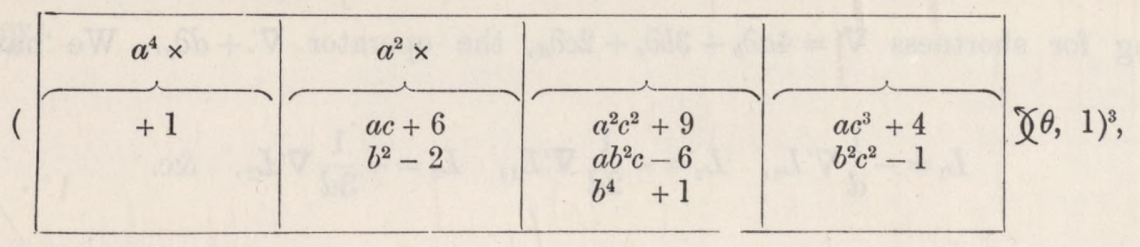

where all the coefficients except the last are reduced to zero by the operator

$$
3 a \partial_{b}+2 b \partial_{c}+c \partial_{d}
$$


and they are consequently (without any alteration) coefficients of the equation of differences of the cubic equation: the last coefficient is not reduced to zero by the operator, and requires therefore to be completed by the adjunction of the terms in $d$ (the series, here and in every other case, is of course a finite one, the number of terms might easily be calculated $\grave{a}$ priori). Let the value be $L_{0}+L_{1} d+L_{2} d^{2}+\&$ c., we have $\dot{L}_{0}=+4 a c^{3}-1 b^{2} c^{2}$; and putting for shortness $\nabla^{\prime}=3 a \partial_{b}+2 b \partial_{c}$, the operator which reduces this to zero is $\nabla^{\prime}+c \partial_{d}$; we ought therefore to have

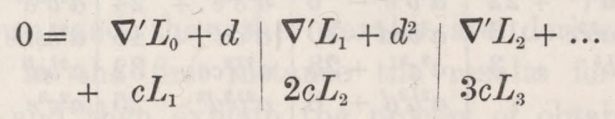

and consequently

$$
L_{1}=-\frac{1}{c} \nabla^{\prime} L_{0}, L_{2}=-\frac{1}{2 c} \nabla^{\prime} L_{1}, L_{3}=-\frac{1}{3 c} \nabla^{\prime} L_{2}, \& c .
$$

giving

$$
\begin{aligned}
& L_{1}=-\frac{1}{c}\left\{(-6+24=) 18 a b c^{2}-4 b^{3} c\right\}=-18 a b c+4 b^{3}, \\
& L_{2}=-\frac{1}{2 c}\left\{-54 a^{2} c+(36-36=) 0 a b^{2}\right\}=+27 a^{2}, \\
& L_{3}=0, \& c .
\end{aligned}
$$

and consequently for the last coefficient the value above written down; it will be presently seen how in more complicated cases the calculations should be arranged.

Again, multiplying together the equation of differences and the equation for the squares of the roots of the cubic equation, we obtain an equation which it is not necessary to write down, as it can be at once formed by putting $e=0$ in the equation of differences for the quartic equation. And from the equation so obtained, by the adjunction of the terms in $e$, we find the equation of differences for the quartic equation, viz. each coefficient is of the form $L_{0}+L_{1} e+L_{2} e^{2}+\& c$., where $L_{0}$ is known, and such coefficient is reduced to zero by the operator

$$
4 a \partial_{b}+3 b \partial_{c}+2 c \partial_{d}+d \partial_{e}
$$

or putting for shortness $\nabla^{\prime}=4 a \partial_{b}+3 b \partial_{c}+2 c \partial_{d}$, the operator $\nabla^{\prime}+d \partial_{e}$. We have therefore

$$
L_{1}=-\frac{1}{d} \nabla^{\prime} L_{0}, \quad L_{2}=-\frac{1}{2 d} \nabla^{\prime} L_{1}, \quad L_{3}=-\frac{1}{3 d} \nabla^{\prime} L_{2}, \quad \& c .
$$

It is to be observed that the last coefficient of the equation of differences is the discriminant, and that the above method of calculating the coefficients of the equation of differences, as applied to the last coefficient, is nothing else than the method of calculating the discriminant given in my Fourth Memoir on Quantics, [155]. 
The multiplication of the equation of differences, and the equation for the squares of the roots of the quartic equation, gives, in like manner, the equation of differences for the quintic equation, except as to the terms involving $f$; and these are obtained as above, viz. each coefficient is of the form $L_{0}+L_{1} f+L_{2} f^{2}+\&$ c., where $L_{0}$ is known; such coefficient is reduced to zero by the operator

$$
5 a \partial_{b}+4 b \partial_{c}+3 c \partial_{d}+2 d \partial_{e}+e \partial_{f}
$$

or putting for shortness $\nabla^{\prime}=5 a \partial_{b}+4 b \partial_{c}+3 c \partial_{d}+2 d \partial_{e}$, the operator $\nabla^{\prime}+e \partial_{f}$. We have therefore

$$
L_{1}=-\frac{1}{e} \nabla^{\prime} L_{0}, \quad L_{2}=-\frac{1}{2 e} \nabla^{\prime} L_{1}, \quad L_{3}=-\frac{1}{3 e} \nabla^{\prime} L_{2}, \quad \& c .
$$

which give $L_{1}, L_{2}$, \&c. by means of $L_{0}$. For the calculation of $\nabla^{\prime} L$ (where $L$ is any one of the coefficients $L_{0}, L_{1}, \&$ c.), it is proper to separate the terms involving the different powers of $e$, and write $L=M_{0}+M_{1} e+M_{2} e^{2}+\&$ c., $\quad \nabla^{\prime}=\nabla^{\prime \prime}+2 d \partial_{e}$, where $\nabla^{\prime \prime}=5 a \partial_{b}+4 b \partial_{c}+3 c \partial_{d}$. We have then

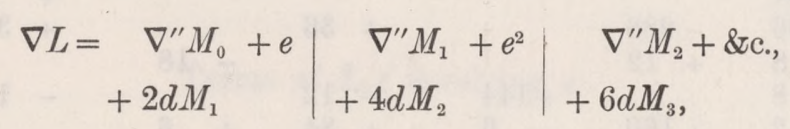

or, what is the same thing,

$$
\begin{array}{rl|l}
\frac{1}{e} \nabla L= & \nabla^{\prime \prime} M_{1}+e & \nabla^{\prime \prime} M_{2}+\ldots \\
+4 d M_{2} & +4 d M_{3}
\end{array}
$$

and as an equation which should be satisfied identically, and which would therefore serve as a verification,

$$
\nabla^{\prime \prime} M_{0}+2 d M_{1}=0
$$

but, since a verification was obtained by other means, the equations of this kind were not used for the purpose. It may be interesting to give the actual calculation of one of the coefficients, say of coefficient $\theta^{2}$ (which, with coefficient $\theta$, was calculated by Mr Harley). 
Calculation of coefficient $\theta^{2}$ in equation of differences for the quintic equation

$$
\left(a, b, c, d, e, f^{\gamma} v, 1\right)^{5}=0
$$

Calculation of $L_{0}$.

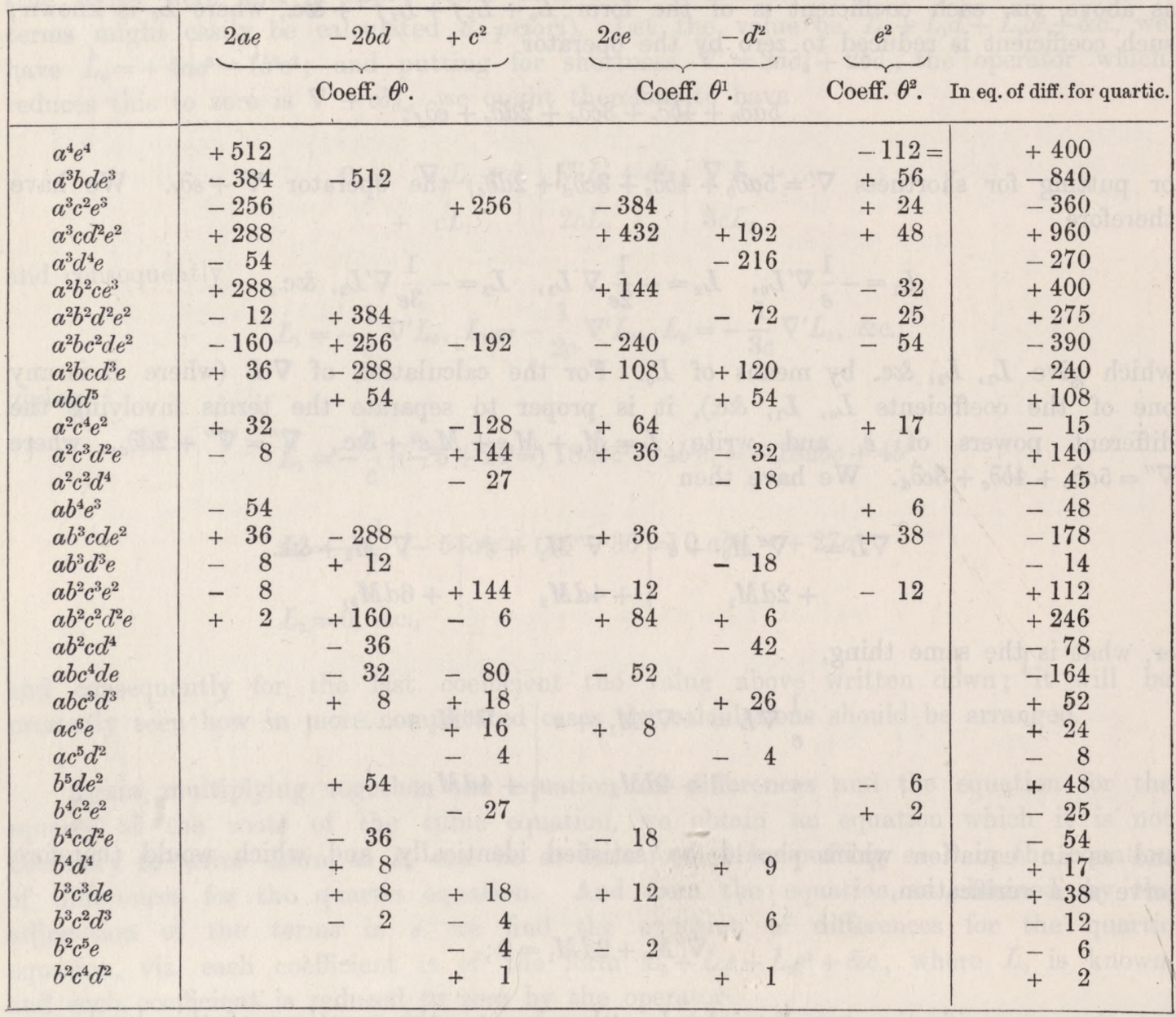

$L_{0}=A+B e+C e^{2}+D e^{3}+E e^{4}$, suppose, then

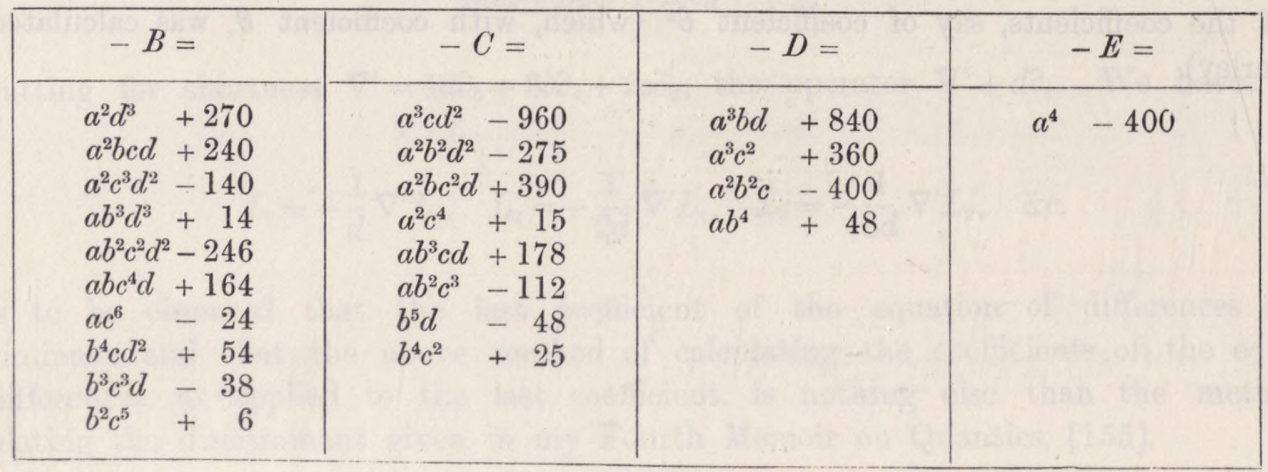


Calculation of $L_{1}$.

Terms of $L_{1} f$ not involving $e$.

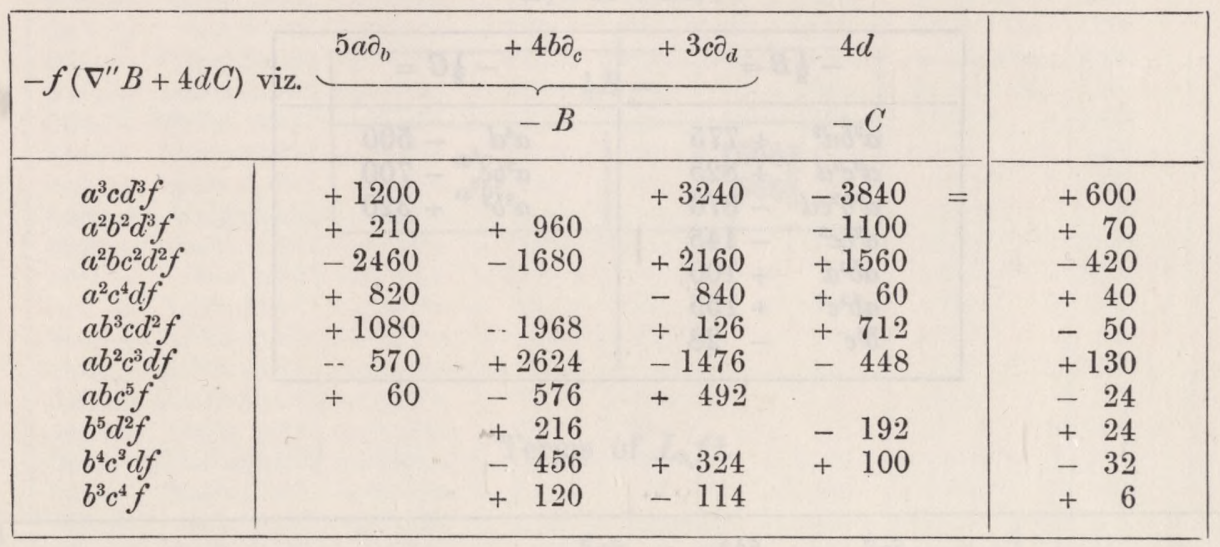

Terms of $L_{1} f$ involving $e^{1}$.

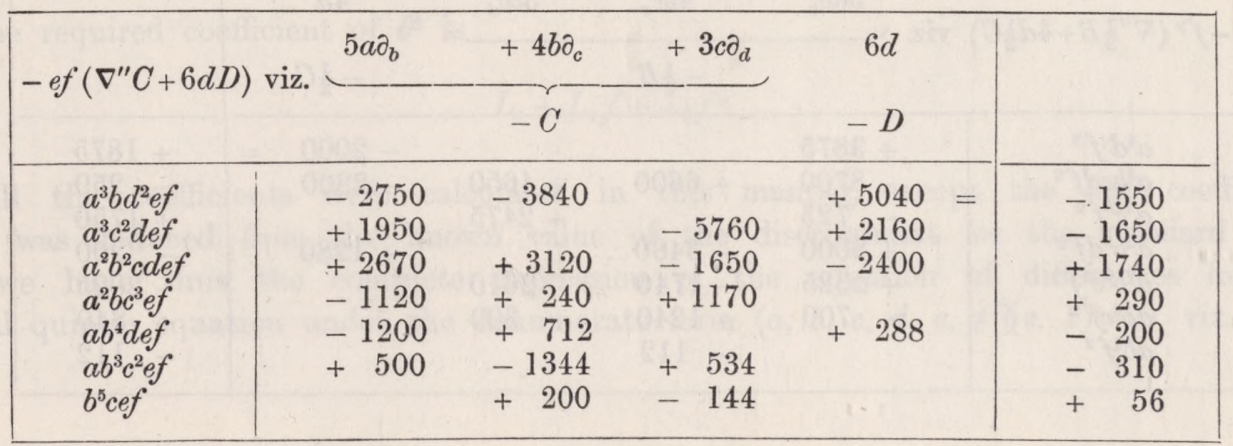

Terms of $L_{1} f$ involving $e^{2}$.

\begin{tabular}{|c|c|c|c|c|c|}
\hline$-e^{2} f\left(\nabla^{\prime \prime} D+\delta\right.$ & $5 a \partial_{b}$ & $\underbrace{4 b \partial_{c}}_{-D}$ & $3 c \partial_{d}$ & $\begin{array}{r}8 d \\
-E\end{array}$ & \\
\hline $\begin{array}{l}a^{4} d e^{2} f \\
a^{3} b c e^{2} f \\
a^{2} b^{3} e^{2} f\end{array}$ & $\begin{array}{l}+4200 \\
-4000 \\
+\quad 960\end{array}$ & $\begin{array}{r} \\
+2880 \\
-1600\end{array}$ & +2520 & $-3200=$ & $\begin{array}{l}+1000 \\
+1400 \\
-\quad 640\end{array}$ \\
\hline
\end{tabular}



ON THE EQUATION OF DIFFERENCES FOR AN EQUATION OF ANY ORDER. [262

Calculation of $L_{2}$.

$$
L_{1}=A+B e+C e^{2} \text {, suppose, where }
$$

\begin{tabular}{|c|c|c|c|}
\hline \multicolumn{2}{|c|}{$-\frac{1}{2} B=$} & \multicolumn{2}{|c|}{$-\frac{1}{2} C=$} \\
\hline $\begin{array}{l}a^{3} b d^{2} \\
a^{3} c^{2} d \\
a^{2} b^{2} c d \\
a^{2} b c^{3} \\
a b^{4} d \\
a b^{3} c^{2} \\
b^{5} c\end{array}$ & $\begin{array}{l}+775 \\
+825 \\
-870 \\
-145 \\
+100 \\
+155 \\
-\quad 28\end{array}$ & $\begin{array}{l}a^{4} d \\
a^{3} b c \\
a^{2} b^{3}\end{array}$ & $\begin{array}{r}-500 \\
-700 \\
+320\end{array}$ \\
\hline
\end{tabular}

Terms of $L_{2} f^{2}$ not involving $e$.

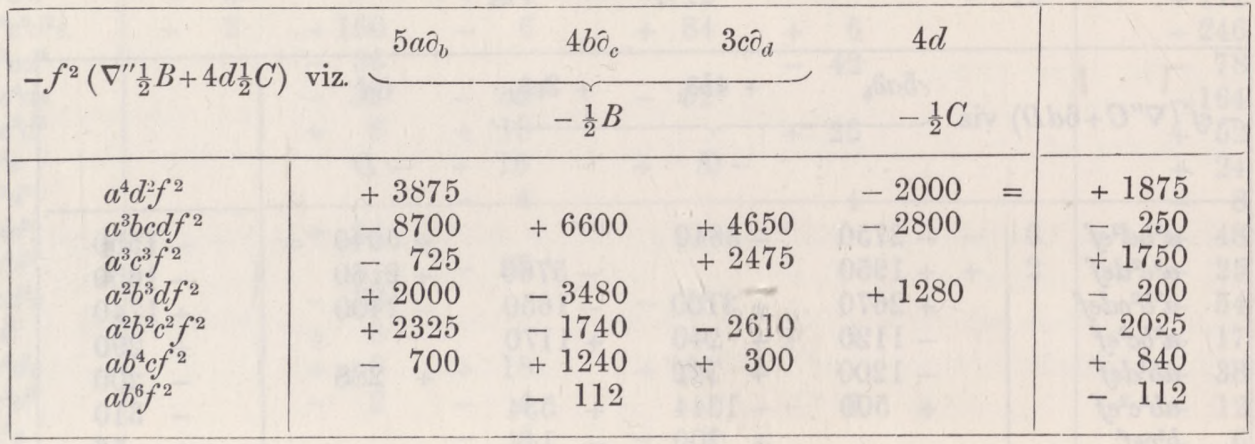

Terms of $L_{2} f^{2}$ involving $e^{1}$.

\begin{tabular}{|l|rrr|r|}
\hline$-e f^{2} \Delta^{\prime \prime} \frac{1}{2} C$ & viz. $\underbrace{5 a \partial_{b}}$ & $\underbrace{4 b_{d c}}_{d c}$ & $3 c \partial_{d}$ \\
& & $-\frac{1}{2} C$ & \\
\hline$a^{4} c e f^{2}$ & -3500 & & -1500 \\
$a^{3} b^{2} e f^{2}$ & +4800 & -2800 & & \\
\hline
\end{tabular}


Calculation of $L_{2}$.

$$
L_{1}=A+B e+C e^{2} \text {, suppose, where }
$$

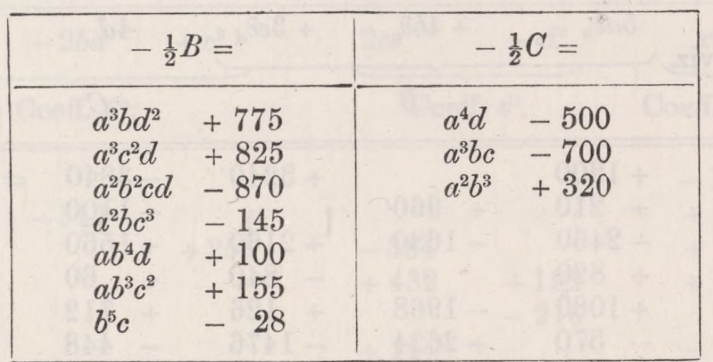

Terms of $L_{2} f^{2}$ not involving $e$.

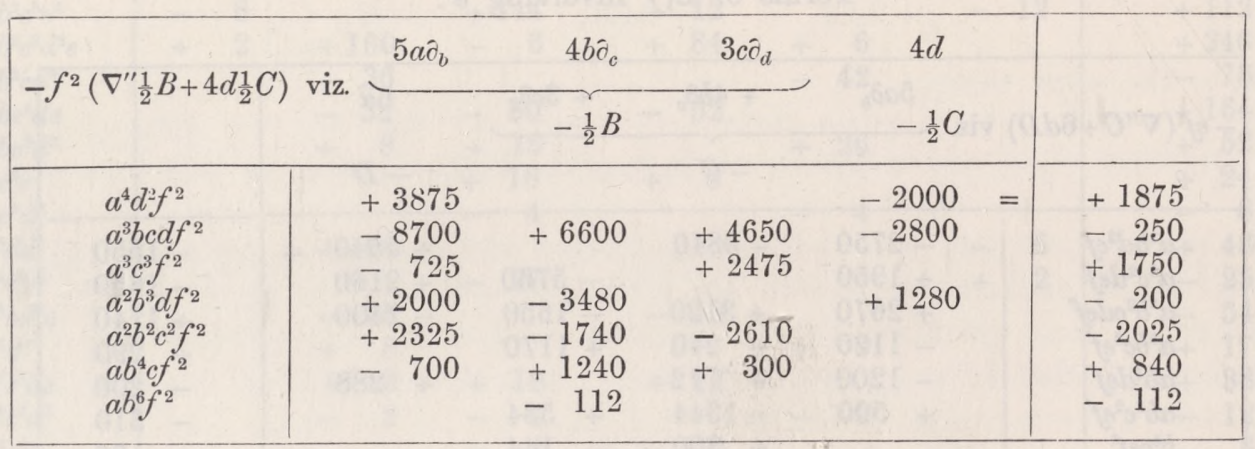

Terms of $L_{2} f^{2}$ involving $e^{1}$.

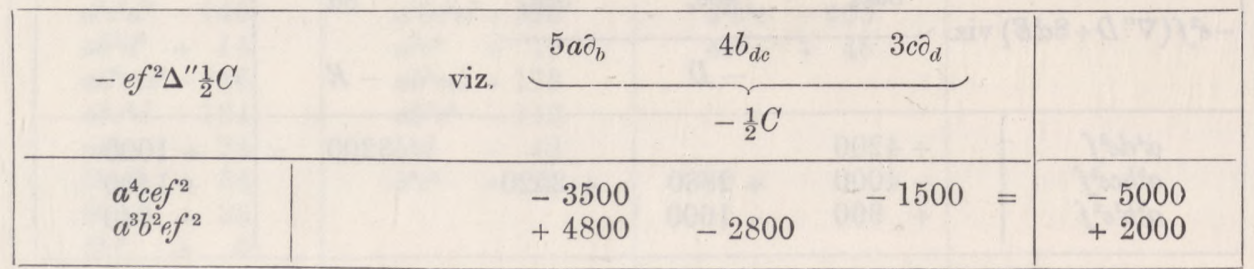


Calculation of $L_{3}$ (gives $L_{3}=0$ ).

$$
L_{2}=A+B e,
$$

\begin{tabular}{|l|l|}
\hline \multicolumn{3}{|c|}{$-\frac{1}{3} B=$} \\
\hline$a^{4} c$ & $+1666 \frac{2}{3}$ \\
$a^{3} b^{2}$ & $-666 \frac{2}{3}$ \\
\hline
\end{tabular}

Terms of $L_{3} f^{3}$.

\begin{tabular}{|c|c|c|c|c|c|}
\hline$-f^{3} \nabla^{\prime \prime \frac{1}{3}} B$ & $5 a \hat{\partial}_{b}$ & $\underbrace{4 b \partial_{c}}_{-\frac{1}{3} B}$ & $3 c \partial_{d}$ & & \\
\hline$a^{4} b f^{3}$ & $-6666 \frac{2}{3}$ & & $+6666_{3}^{2}$ & $=$ & 0 \\
\hline
\end{tabular}

and the required coefficient of $\theta^{2}$ is

$$
L_{0}+L_{1} f+L_{2} f^{2} .
$$

All the coefficients were calculated in this manner, except the last coefficient, which was deduced from the known value of the discriminant for the standard form. And we have thus the complete expression of the equation of differences for the general quintic equation under the denumerate form $\left(a, b, c, d, e, f^{r}(v, 1)^{5}=0\right.$, viz.

C. IV. 
Equation of differences for $\left(a, b, c, d, e f^{*} v, 1\right)^{5}=0$ is

\begin{tabular}{|c|c|c|c|c|c|c|c|}
\hline$\theta^{10}$ & $\theta^{9}$ & $\theta^{8}$ & $\theta^{7}$ & $\theta^{6}$ & $\theta^{5}$ & & $\theta^{4}$ \\
\hline$a^{8} \times$ & $a^{6} \times$ & $a^{4} \times$ & $a^{2} \times$ & & 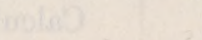 & & \\
\hline+1 & $\begin{array}{l}a c+10 \\
b^{2}-4\end{array}$ & $\begin{array}{l}a^{3} e+10 \\
a^{2} b d-4 \\
a^{2} c^{2}+39 \\
a b^{2} c-30 \\
b^{4}+6\end{array}$ & $\begin{array}{l}a^{4} c e+50 \\
a^{4} d^{2}+25 \\
a^{3} b^{2} e-20 \\
a^{3} b c d-50 \\
a^{3} c^{3}+80 \\
a^{2} b^{3} d+16 \\
a^{2} b^{2} c^{2}-81 \\
a b^{4} c+30 \\
b^{6}-4\end{array}$ & $\begin{array}{l}a^{6} d f+200 \\
a^{6} e^{2}-95 \\
a^{5} b c f-120 \\
a^{5} b d e+36 \\
a^{5} c^{2} e+124 \\
a^{5} c d^{2}+92 \\
a^{4} b^{3} f+32 \\
a^{4} b^{2} c e-98 \\
a^{4} b^{2} d^{2}-44 \\
a^{4} b c^{2}-160 \\
a^{4} c^{4}+95 \\
a^{3} b^{4}++18 \\
a^{3} b^{3} c d+116 \\
a^{3} b^{2} c^{3}-104 \\
a^{2} b^{5} d-20 \\
a^{2} b^{4} c^{2}+45 \\
a b^{6} c^{2}-10 \\
b^{8}+1\end{array}$ & $\begin{array}{l}a^{6} f^{2}+625 \\
a^{5} b e f-250 \\
a^{5} c d f+400 \\
a^{5} c e^{2}-360 \\
a^{5} d^{2} e+260 \\
a^{4} b^{2} d f-110 \\
a^{4} b^{2} e^{2}+169 \\
a^{4} b c^{2} f-240 \\
a^{4} b c d e-104 \\
a^{4} b d^{3}-104 \\
a^{4} c^{3} e+196 \\
a^{4} c^{2} d^{2}+118 \\
a^{3} b^{3} c f+150 \\
a^{3} b^{3} d e-10 \\
a^{3} b^{2} c^{2} e-180 \\
a^{3} b^{2} c d^{2}+20 \\
a^{3} b c^{3} d-220 \\
a^{3} c^{5}+66 \\
a^{2} b^{5} f-24 \\
a^{2} b^{4} c e+66 \\
a^{2} b^{4} d^{2}-3 \\
a^{2} b^{3} c^{2} d+192 \\
a^{2} b^{2} c^{4}-66 \\
a b^{6} e-8 \\
a l^{5} c d-66 \\
a b^{4} c^{3}+24 \\
b^{7} d+8 \\
b^{6} c^{2}-3\end{array}$ & $\begin{array}{l}a^{5} c f^{2} \\
a^{5} d e f \\
a^{5} e^{3} \\
a^{4} b^{2} f^{2} \\
a^{4} b c e f \\
a^{4} b d^{2} f \\
a^{4} b d e^{2} \\
a^{4} c^{2} d f \\
a^{4} c^{2} e^{2} \\
a^{4} c d^{2} e \\
a^{4} d^{4} \\
a^{3} b^{3} e f \\
a^{3} b^{2} c e^{2} \\
a^{3} b^{2} c d f \\
a^{3} b^{2} d^{2} e \\
a^{3} b c^{3} f \\
a^{3} b c^{2} d e- \\
a^{3} b c d^{3} \\
a^{3} c^{4} e \\
a^{3} c^{3} d^{2} \\
a^{2} b^{4} d f \\
a^{2} b^{4} e^{2} \\
a^{2} b^{3} c^{2} f+ \\
a^{2} b^{3} c d e \\
a^{2} b^{3} d^{2} \\
a^{2} b^{2} c^{3} e \\
a^{2} b^{2} c^{2} d^{2}+ \\
a^{2} b c^{4} d \\
a^{2} c^{6} \\
a b^{5} c f \\
a b^{5} d e \\
a b^{4} c^{2} e+ \\
a b^{4} c d^{2}- \\
a b^{3} c^{3} d+ \\
a b^{2} c^{5} \\
b^{7} f \\
b^{6} c e \\
b^{6} d^{2} \\
b^{5} c^{2} d \\
b^{4} c^{4}\end{array}++$ & $\begin{array}{rr}+1750 \\
-\quad 950 \\
+\quad 40 \\
-\quad 700 \\
-\quad 130 \\
+\quad 380 \\
+\quad 142 \\
+\quad 240 \\
-\quad 522 \\
+\quad 708 \\
-\quad 53 \\
+\quad 128 \\
+\quad 388 \\
-\quad 394 \\
-\quad 378 \\
-\quad 144 \\
-\quad 480 \\
-\quad 156 \\
+\quad 194 \\
+\quad 52 \\
+\quad 66 \\
-\quad 84 \\
+\quad 194 \\
+\quad 330 \\
+\quad 92 \\
-\quad 152 \\
+\quad 174 \\
-\quad 140 \\
+\quad 25 \\
-\quad 70 \\
-\quad 42 \\
+\quad 32 \\
-\quad 144 \\
+\quad 100 \\
-\quad 18 \\
+ & 8 \\
- & 2 \\
+ & 22 \\
- & 16 \\
+ & 3\end{array}$ \\
\hline
\end{tabular}


$(8 \theta, 1)^{10}=0$, viz. the function in $\theta$ is

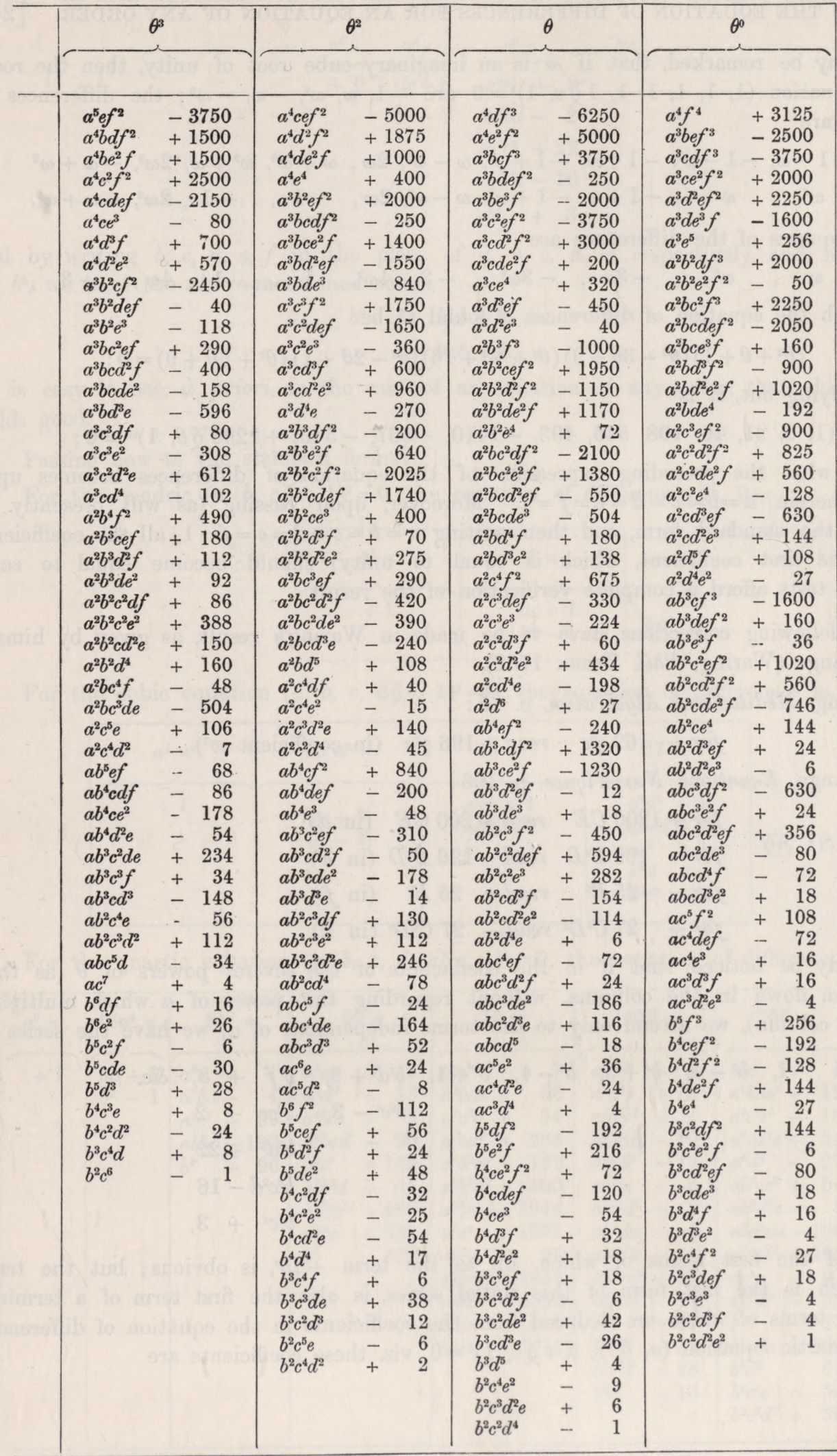


It may be remarked, that if $\omega$ is an imaginary cube root of unity, then the roots of the equation $(1,1,1,1,1,1 \gamma v, 1)^{5}=0$ are $-1, \omega, \omega^{2},-\omega,-\omega^{2}$; the differences of the roots are

$$
\begin{aligned}
& -1-\omega,-1-\omega^{2},-1+\omega,-1+\omega^{2}, \omega-\omega^{2}, 2 \omega, \omega+\omega^{2}, \omega^{2}+\omega, 2 \omega^{2},-\omega+\omega^{2} \\
= & \omega^{2}, \omega,-1+\omega,-1+\omega^{2}, \omega-\omega^{2}, 2 \omega,-1,-1,2 \omega^{2},-\omega+\omega^{2}
\end{aligned}
$$

and the squares of the differences are

$$
\omega, \quad \omega^{2},-3 \omega,-3 \omega^{2},-3,4 \omega^{2}, 1,1,4 \omega,-3,
$$

from which the equation of differences is found to be

$$
\left(\theta^{2}+\theta+1\right)\left(\theta^{2}-3 \theta+9\right)\left(\theta^{2}+4 \theta+16\right)\left(\theta^{2}-2 \theta+1\right)\left(\theta^{2}+6 \theta+9\right)=0 ;
$$

or multiplying out, it is

$$
\left(1,6,21,46,108,546,493,-1410,-567,-540,+1296 \gamma(\theta, 1)^{10}=0 ;\right.
$$

which is what the preceding expression of the equation of differences becomes upon writing therein $a=b=c=d=e=f=1$. Moreover, upon passing (as will presently be done) to the standard form, and then writing $a=b=c=d=e=f=1$, all the coefficients (except the first coefficient, which is equal to unity) should become equal to zero; these two tests afford a complete verification of the result.

The following corrections have to be made in Waring's result, as given by himself and Lagrange (Waring, Phil. Trans. 1762).

Waring, Meditationes Algebraicae, p. 85 :

$$
\text { for }+169 q^{3} s \quad \text { read }+196 q^{3} s \text { (in coefficient } w^{5} \text { ). }
$$

Lagrange, Equations Numériques, p. 108 :

$$
\begin{aligned}
& \text { for }+1200 C E \text { read }+200 C E \text { (in } d \text { ) } \\
& \text { for - } 169 B^{3} D \text { read }-196 B^{3} D \text { (in } e \text { ) } \\
& \text { for }-25 B^{6} \quad r e a d+25 B^{6} \text { (in } f \text { ) } \\
& \text { for }+27 C^{4} D^{2} \text { read }-27 C^{4} D^{2} \text { (in } k \text { ). }
\end{aligned}
$$

It may be noticed, that if in the coefficients of the several powers of $\theta$ (as they are written down in the columns, without regarding the power of $a$ which multiplies the entire column), we attend only to the terms independent of $a$, we have the series

$$
\begin{aligned}
& 1, \quad b^{2}-4, \quad b^{4}+6, \quad b^{6}-4, \quad b^{8}+1, \quad b^{7} d+8, \quad b^{7} f+8, \quad \& c . \\
& b^{6} c^{2}-3, \quad b^{6} c e-2 \\
& b^{6} d^{2}+22 \\
& b^{5} c^{2} d-16 \\
& b^{4} c^{4}+3,
\end{aligned}
$$

the law of the first terms of which, up to the term $+1 b^{8}$, is obvious; but the term $+1 b^{8}$, which is the last term of this initial series, is also the first term of a terminal series, the terms of which are deduced from the coefficients in the equation of differences for the quartic equation $\left(a, b, c, d e^{\gamma}(v, 1)^{4}=0\right.$, viz. these coefficients are 
It may be remarked, that if $\omega$ is an imaginary cube root of unity, then the roots of the equation $(1,1,1,1,1,1) v, 1)^{5}=0$ are $-1, \omega, \omega^{2},-\omega,-\omega^{2}$; the differences of the roots are

$$
\begin{aligned}
& -1-\omega,-1-\omega^{2},-1+\omega,-1+\omega^{2}, \omega-\omega^{2}, 2 \omega, \omega+\omega^{2}, \omega^{2}+\omega, 2 \omega^{2},-\omega+\omega^{2} \\
= & \omega^{2}, \omega,-1+\omega,-1+\omega^{2}, \omega-\omega^{2}, 2 \omega,-1,-1,2 \omega^{2},-\omega+\omega^{2},
\end{aligned}
$$

and the squares of the differences are

$$
\omega, \omega^{2},-3 \omega,-3 \omega^{2},-3,4 \omega^{2}, 1,1,4 \omega,-3,
$$

from which the equation of differences is found to be

$$
\left(\theta^{2}+\theta+1\right)\left(\theta^{2}-3 \theta+9\right)\left(\theta^{2}+4 \theta+16\right)\left(\theta^{2}-2 \theta+1\right)\left(\theta^{2}+6 \theta+9\right)=0
$$

or multiplying out, it is

$$
(1,6,21,46,108,546,493,-1410,-567,-540,+1296 \gamma \theta, 1)^{10}=0 ;
$$

which is what the preceding expression of the equation of differences becomes upon writing therein $a=b=c=d=e=f=1$. Moreover, upon passing (as will presently be done) to the standard form, and then writing $a=b=c=d=e=f=1$, all the coefficients (except the first coefficient, which is equal to unity) should become equal to zero; these two tests afford a complete verification of the result.

The following corrections have to be made in Waring's result, as given by himself and Lagrange (Waring, Phil. Trans. 1762).

Waring, Meditationes Algebraicce, p. 85 :

$$
\text { for }+169 q^{3} s \quad r e a d+196 q^{3} s \text { (in coefficient } w^{5} \text { ). }
$$

Lagrange, Equations Numériques, p. 108 :

$$
\begin{aligned}
& \text { for }+1200 C E \text { read }+200 C E \text { (in } d \text { ) } \\
& \text { for }-169 B^{3} D \text { read }-196 B^{3} D \text { (in } e \text { ) } \\
& \text { for }-25 B^{6} \quad \text { read }+25 B^{6} \text { (in } f \text { ) } \\
& \text { for }+27 C^{4} D^{2} \text { read }-27 C^{4} D^{2} \text { (in } k \text { ). }
\end{aligned}
$$

It may be noticed, that if in the coefficients of the several powers of $\theta$ (as they are written down in the columns, without regarding the power of $a$ which multiplies the entire column), we attend only to the terms independent of $a$, we have the series

$$
\begin{aligned}
& 1, \quad b^{2}-4, \quad b^{4}+6, \quad b^{6}-4, \quad b^{8}+1, \quad b^{7} d+8, \quad b^{7} f+8, \quad \& c . \\
& b^{6} c^{2}-3, \quad b^{6} c e-2 \\
& b^{6} d^{2}+22 \\
& b^{5} c^{2} d-16 \\
& b^{4} c^{4}+3 \text {, }
\end{aligned}
$$

the law of the first terms of which, up to the term $+1 b^{8}$, is obvious; but the term $+1 b^{8}$, which is the last term of this initial series, is also the first term of a terminal series, the terms of which are deduced from the coefficients in the equation of differences for the quartic equation $\left(a, b, c, d e^{\gamma}(v, 1)^{4}=0\right.$, viz. these coefficients are 


$$
\begin{aligned}
& a^{6} \times \quad a^{4} \times \quad a^{2} \times \quad \& \\
& \sim+1 \overbrace{a c+8}^{a^{2} \times} \overbrace{a^{3} e+8}^{a^{2} \times} \\
& b^{2}-3 \quad a^{2} b d-2 \\
& a^{2} c^{2}+22 \\
& a b^{2} c-16 \\
& b^{4}+3 \text {; }
\end{aligned}
$$

\&c.

and by writing $b, c, d, e, f$ in the place of $a, b, c, d, e$ respectively, and multiplying by $b^{2}$, we have the above-mentioned series,

$$
\begin{array}{cc}
b^{8}+1, & b^{7} d+8, \& c . \\
& b^{6} c^{2}-3 .
\end{array}
$$

\begin{tabular}{|c|c|c|c|}
\hline$a^{4} \times$ & $18 a^{2} \times$ & $81 \times$ & $27 \times$ \\
\hline+1 & $\begin{array}{l}a c+1 \\
b^{2}-1\end{array}$ & $\begin{array}{l}a^{2} c^{2}+1 \\
a b^{2} c-2 \\
b^{4}+1\end{array}$ & $\begin{array}{l}a^{2} d^{2}+1 \\
a b c d-6 \\
a c^{3}+4 \\
b^{3} d+4 \\
b^{2} c^{2}-3\end{array}$ \\
\hline
\end{tabular}

It is easy to see, $\grave{c}$ priori, in the case of an equation of any order, that this property

\begin{tabular}{|c|c|c|c|c|c|c|c|c|}
\hline$a^{6} \times$ & & $8 a^{2} \times$ & & $2 x$ & $16 \times$ & $1152 \times$ & $256 \times$ & \\
\hline+1 & $\begin{array}{l}a c+1 \\
b^{2}-1\end{array}$ & $\begin{array}{l}a^{3} e+1 \\
a^{2} b d-4 \\
a^{2} c^{2}+99 \\
a b^{2} c-192 \\
b^{4}+96\end{array}$ & $\begin{array}{l}a^{4} c e \\
a^{4} d^{2} \\
a^{3} b^{2} e \\
a^{3} b c d \\
a^{3} c^{3} \\
a^{2} b^{3} d \\
a^{2} b^{2} c^{2} \\
a b^{4} c \\
b^{6}\end{array}$ & $\begin{array}{l}+3 \\
+\quad 13 \\
-\quad 3 \\
-\quad 90 \\
+\quad 189 \\
+\quad 64 \\
-432 \\
+384 \\
-128\end{array}$ & $\begin{array}{l}a^{4} e^{2}-7 \\
a^{3} b d e+56 \\
a^{3} c^{2} e+54 \\
a^{3} c d^{2}+288 \\
a^{2} b^{2} c e-192 \\
a^{2} b^{2} d^{2}-400 \\
a^{2} b c^{2} d-1944 \\
a^{2} c^{4}+1377 \\
a b^{4} e+96 \\
a b^{3} c d+3648 \\
a b^{2} c^{3}-2592 \\
b^{5} d-1536 \\
b^{4} c^{2}+1152\end{array}$ & $\begin{array}{l}a^{3} c e^{2}-1 \\
a^{3} d^{2} e+3 \\
a^{2} b^{2} e^{2}+1 \\
a^{2} b c d e-10 \\
a^{2} b d^{3}-12 \\
a^{2} c^{3} e+6 \\
a^{2} c^{2} d^{2}+9 \\
a b^{3} d e+4 \\
a b^{2} c^{2} e-3 \\
a b^{2} c d^{2}+56 \\
a b c^{3} d-78 \\
a c^{5}+27 \\
b^{4} d^{2}-32 \\
b^{3} c^{2} d+48 \\
b^{2} c^{2}-18\end{array}$ & $\begin{array}{l}a^{3} e^{3}+1 \\
a^{2} b d e^{2}-12 \\
a^{2} c^{2} e^{2}-18 \\
a^{2} c d^{2} e+54 \\
a^{2} d^{4}-27 \\
a b^{2} c e^{2}+54 \\
a b^{2} d^{2} e-6 \\
a b c^{2} d e-180 \\
a b c d^{3}+108 \\
a c^{4} e+81 \\
a c^{3} d^{2}-54 \\
b^{4} e^{2}-27 \\
b^{3} c d e+108 \\
b^{3} d^{3}-64 \\
b^{2} c^{3} e-54 \\
b^{2} c^{2} d^{2}+36\end{array}$ & $\gamma \theta, 1)^{6}$ \\
\hline
\end{tabular}
holds good.

Passing now to the standard forms:

For the quadric $(a, b, c \gamma v, 1)^{2}=0$, the equation of differences is, $0=$

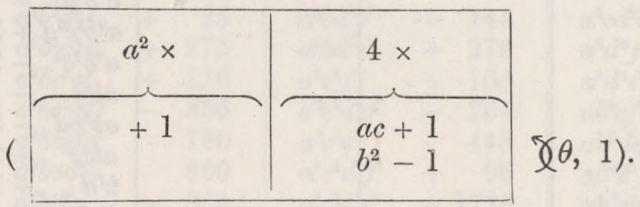

For the cubic equation $(a, b, c, d \gamma v, 1)^{3}=0$, the equation of differences is, $0=$

For the quartic equation $\left(a, b, c, d, e^{\gamma} v, 1\right)^{4}=0$, the equation of differences is, $0=$ 
For the quintic equation $(a, b, c, d, e, f \gamma v, 1)^{5}=0$, the equation of differences is,

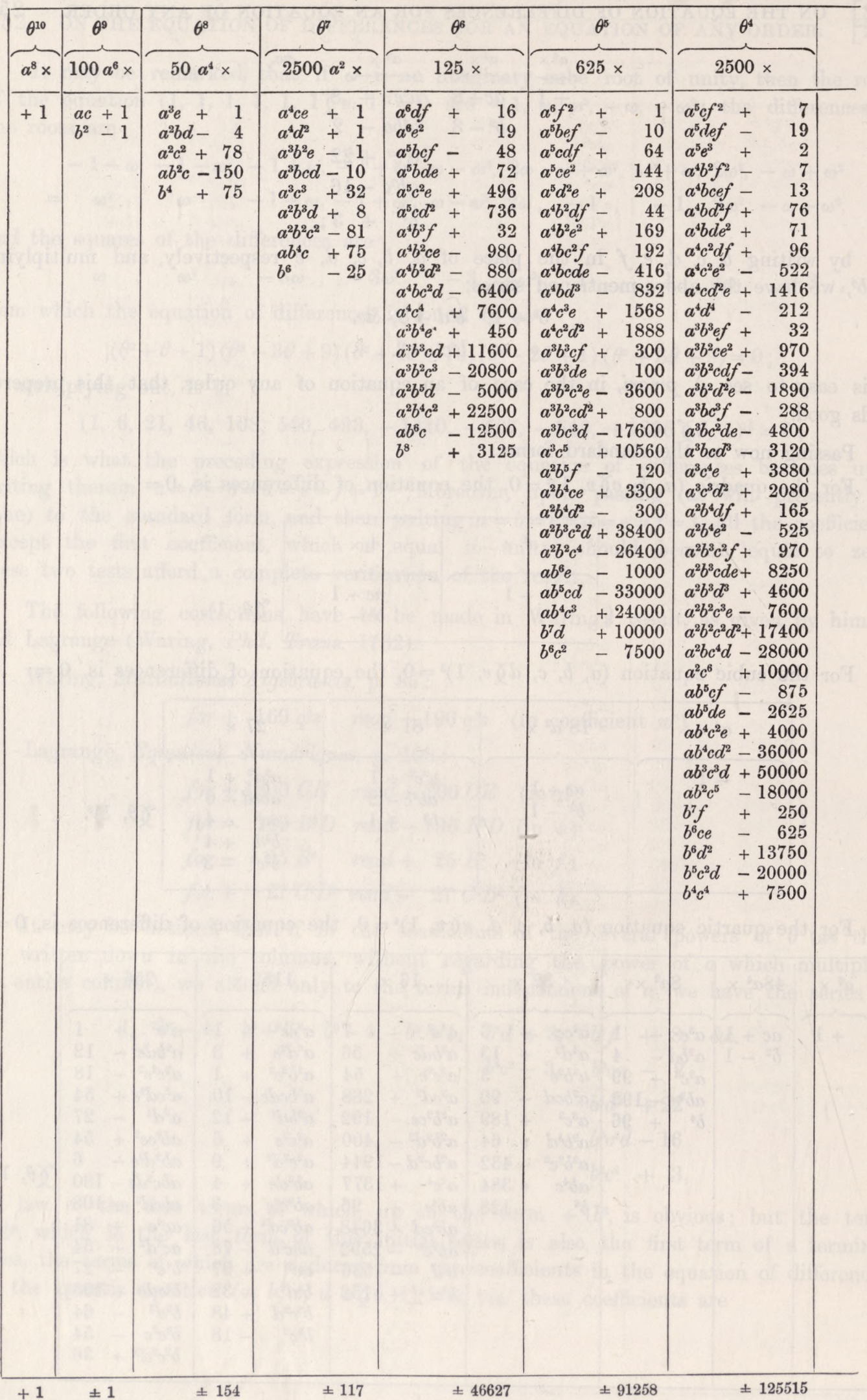


$0=\left({ }^{\gamma} \theta, 1\right)^{10}=0$, viz. the function in $\theta$ is

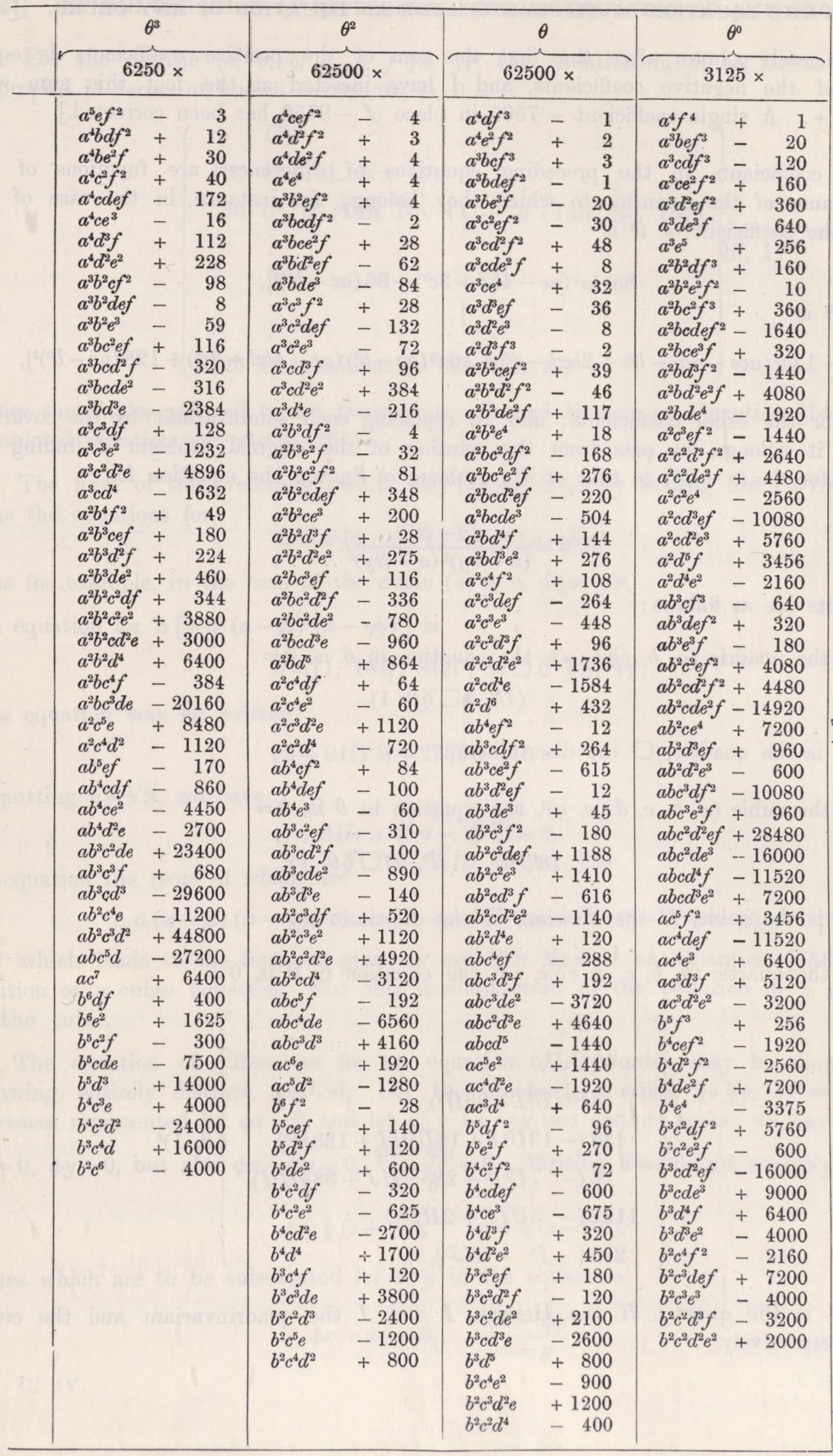


[where in each column after the first the sum of the positive coefficients is equal to that of the negative coefficients, and I have inserted at the foot this sum with the sign + . A single coefficient -7500 in place of -3750 has been corrected.]

The coefficients in the preceding equations of differences are functions of the seminvariants of the quantics to which they belong; for instance, in the case of the quartic, the coefficient of $\theta^{4}$ is

that of $\theta^{3}$ is

$$
8 a^{2}\left\{a^{2}\left(a e-4 b d+3 c^{2}\right)+96\left(a c-b^{2}\right)^{2}\right\},
$$

$$
32\left\{-13 a^{3}\left(a c e-a d^{2}-b^{2} e+2 b c d-c^{3}\right)+16 a^{2}\left(a c-b^{2}\right)\left(a e-4 b d+3 c^{2}\right)+128\left(a c-b^{2}\right)^{3}\right\},
$$

and so for the other coefficients; and by replacing each seminvariant by the covariant to which it belongs, we pass from the solution of the original problem of finding the equation for $\theta=(\alpha-\beta)^{2}$, to that of the problem of finding the equation for

$$
\theta=\frac{(x-\beta)^{2}}{(x-\alpha y)^{2}(x-\beta y)^{2}} .
$$

The results are as follows:

For the quadric $\left(a, b, c \bigvee(x, y)^{2}\right.$, the equation in $\theta$ is, $0=$

$$
\left(U^{2}, 4 \square \gamma \theta, 1\right),
$$

where $U$ is the quadric, $\square$ the discriminant.

For the cubic $\left(a, b, c, d \gamma(x, y)^{3}\right.$, the equation in $\theta$ is, $0=$

$$
\left(U^{4}, 18 U^{2} H, 81 H^{2}, 27 \square \gamma \theta \theta, 1\right)^{3},
$$

where $U$ is the cubic, $H$ the Hessian, $\square$ the discriminant.

For the quartic $\left(a, b, c, d, e \gamma(x, y)^{4}\right.$, the equation in $\theta$ is, $0=$

$$
\left\{\begin{array}{l}
U^{6}, \\
48 U^{4} H, \\
8 U^{2}\left(U^{2} I+96 H^{2}\right), \\
32\left(-13 U^{3} J+16 U^{2} H I+128 H^{3}\right), \\
16\left(-7 U^{2} I^{2}-288 U H J+384 H^{2} I\right) \\
1152\left(-3 U I J+2 H I^{2}\right) \\
256\left(I^{3}-27 J^{2}\right),
\end{array}\right\}(\theta, 1)^{6},
$$

where $U$ is the quartic, $H$ the Hessian, $I$ and $J$ the quadrinvariant and the cubinvariant respectively. 
For the quintic $\left(a, b, c, d, e, f \gamma(x, y)^{5}\right.$, the equation in $\theta$, as far as it can be expressed in terms of known covariants, is, $0=$

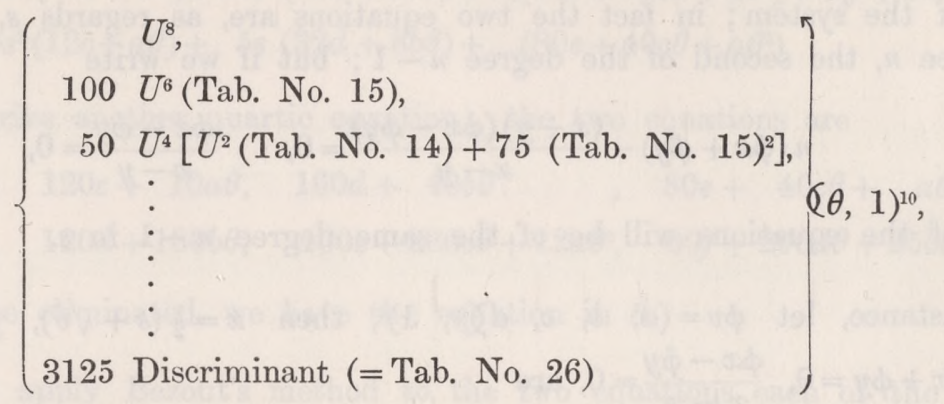

where the Tables referred to are those in my Second Memoir on Quantics, [141; for the completed equation see post p. 261].

The form of the preceding results may be nodified by writing $\theta=9 \div U^{2}$; we have thus the equations for

$$
9=a^{2}(\alpha-\beta)^{2}(x-\gamma y)^{2}(x-\delta y)^{2} \ldots ;
$$

thus for example, in the case of the cubic $\left(a, b, c, d \gamma(x, y)^{3}\right.$,

the equation for $9\left[=a^{2}(\alpha-\beta)^{2}(x-\gamma y)^{2}\right]$ is

$$
0=\left(1,18 H, 81 H^{2}, 27 \square U^{2} \gamma 9,1\right)^{3} ;
$$

this equation may be written

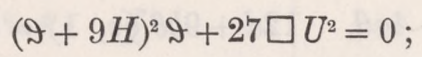

or putting $v=\sqrt{\overline{9}}$, we have

$$
v^{3}+9 H v+U \sqrt{-27 \square}=0,
$$

an equation the roots of which are

$$
a(\alpha-\beta)(x-\gamma y), \quad a(\beta-\gamma)(x-\alpha y), \quad a(\gamma-\alpha)(x-\beta y),
$$

and which leads to the formula, given in my fifth Memoir on Quantics, [156], for the solution of a cubic equation. But this decomposition of the equation in 9 is peculiar to the cubic.

The equation of differences for an equation of any order may be found by the following entirely distinct mett.jd. Let the proposed equation $(* \gamma v, 1)^{n}=0$, be for shortness represented by $\phi v=0$, and let $x, y$ be any two distinct roots; we have not only $\phi x=0, \phi y=0$, but also $\phi x+\phi y=0, \frac{\phi x-\phi y}{x-y}=0$. Writing $\theta=(x-y)^{2}, s=x+y$, we have

$$
x=\frac{1}{2}(s+\sqrt{\theta}), \quad y=\frac{1}{2}(s-\sqrt{\theta}),
$$

values which are to be substituted for $x, y$ in the equations

$$
\phi x+\phi y=0, \quad \frac{\phi x-\phi y}{x-y}=0 .
$$

C. IV. 
We have thus two equations rational in $s$ and $\theta$, and the elimination between them of the quantity $s$ leads to the required equation in $\theta$. But it is proper to modify the form of the system; in fact the two equations are, as regards $s$, the first of them of the degree $n$, the second of the degree $n-1$; but if we write

$$
n(\phi x+\phi y)-\frac{(x+y)(\phi x-\phi y)}{x-y}=0, \quad \frac{\phi x-\phi y}{x-y}=0,
$$

then each of the equations will be of the same degree $n-1$ in $s$.

For instance, let $\phi v=\left(a, b, c, d \gamma(v, 1)^{3}\right.$, then $x=\frac{1}{2}(s+\sqrt{ } \theta), y=\frac{1}{2}(s+\sqrt{\theta})$; the equations $\phi x+\phi y=0, \frac{\phi x-\phi y}{x-y}=0$, are

$$
\begin{aligned}
s^{3} \cdot a+3 s^{2} \cdot 2 b+3 s(4 c+a \theta)+8 d+6 b \theta & =0 \\
3 s^{2} \cdot a+3 s \cdot 4 b+12 c+a \theta & =0 ;
\end{aligned}
$$

and multiplying the first equation by 3 and the second by $-s$, adding and dividing by 2 , we have an equation

$$
s^{2} \cdot 3 b+s(12 c+4 a \theta)+12 d+9 b \theta=0 .
$$

The second equation and this equation may be written

$$
\begin{array}{ll}
(3 a, 12 b & 12 c+a \theta \gamma(s, 1)^{2}=0, \\
(3 b, 12 c+4 a \theta, & 12 d+9 b \theta \gamma(s, 1)^{2}=0,
\end{array}
$$

and the elimination of $s$ from these equations gives the required equation in $\theta$. The result may be obtained under either of the two forms,

$$
\left\{a^{2} \theta^{2}+\left(15 a c-27 b^{2}\right) \theta-36\left(b d-c^{2}\right)\right\}\left\{a^{2} \theta+3\left(a c-b^{2}\right)\right\}+3\{2 a b \theta+3(a d-b c)\}^{2}=0
$$

and

$$
\left\{4 a^{2} \theta^{2}+\left(24 a c-27 b^{2}\right) \theta-36\left(b d-c^{2}\right)\right\}\left\{a^{2} \theta+12\left(a c-b^{2}\right)\right\}+3\{a b \theta+6(a d-b c)\}^{2}=0,
$$

the expansions of which respectively coincide with the before-mentioned result.

In the case of the quartic equation $\phi v=(a, b, c, d \gamma v, 1)^{4}=0$, we have

$$
\begin{aligned}
s^{4} \cdot a+4 s^{3} \cdot 2 b+6 s^{2}(4 c+a \theta)+4 s(8 d+6 b \theta)+16 e+24 c \theta+a \theta^{2} & =0, \\
4 s^{3} \cdot a+6 s^{2} \cdot 4 b+4 s(12 c+a \theta)+32 d+8 b \theta & =0,
\end{aligned}
$$

from which we derive another cubic equation; the two cubic equations thus are

$$
\begin{aligned}
& \left(4 a, 24 b \quad, 48 c+4 a \theta, 32 d+8 b \theta \quad \gamma(s, 1)^{3}=0,\right. \\
& \left(4 b, \quad 24 c+10 a \theta, \quad 48 d+44 b \theta, \quad 32 e+48 c \theta+2 a \theta^{2} \gamma s, 1\right)^{3}=0,
\end{aligned}
$$

from which, if $s$ be eliminated, we have the equation in $\theta$. 
Similarly, for the quintic equation $\phi v=\left(a, b, c, d, e, f \gamma(v, 1)^{5}=0\right.$, we have $s^{5} \cdot a+5 s^{4} \cdot 2 b+10 s^{3}(4 c+a \theta)+10 s^{2}(8 d+6 b \theta)+5 s\left(16 e+24 c \theta+a \theta^{2}\right)+32 f+80 d \theta+10 b \theta^{2}=0$, $5 s^{4} \cdot a+10 s^{3} \cdot 4 b+1 G s^{2}(12 c+a \theta)+5 s(32 d+8 b \theta)+\left(80 e+40 c \theta+a \theta^{2}\right) \quad=0$, from which we derive another quartic equation; the two equations are $(5 a$,

$$
\begin{array}{lllll}
(5 a, & 40 b \quad, & 120 c+10 a \theta, & 160 d+40 b \theta \quad, & 80 e+40 c \theta+a \theta^{2} \gamma(s, 1)^{4}=0, \\
(5 b, & 40 c+20 a \theta, & 120 d+130 b \theta, & 160 e+280 c \theta+12 a \theta^{2}, & 80 f+200 d \theta+25 b \theta^{2} \gamma(s, 1)^{4}=0,
\end{array}
$$

from which, if $s$ be eliminated, we have the equation in $\theta$.

But we may apply Bezout's method to the two equations each of the order $n-1$, which result from the equation of the $n$th order $\phi v=(* \gamma v, 1)^{n}=0$. The process is as follows: Suppose, in general, that $s$ is to be eliminated from the two equations

$$
F s=0, \quad G s=0,
$$

each of the order $n-1$; it is only necessary to form the expression

$$
\frac{F s G s^{\prime}-F s^{\prime} G s}{s-s^{\prime}}
$$

which will be a function of $s, s^{\prime}$ of the form

$$
\left\{\begin{array}{l}
a_{0,0}, a_{0,1}, \ldots a_{0, n-2} \gamma(s, 1)^{n-2}\left(s^{\prime}, 1\right)^{n-2} \\
a_{1,0}, \\
\vdots \\
a_{n-2,0},
\end{array}\right.
$$

where the coefficients are such that $a_{l, m}=a_{m, l}$; and by equating to zero the determinant formed with these coefficients, we have the result of the elimination.

In the present case, writing for a moment $\phi \frac{1}{2}(s+\sqrt{\theta})=A, \phi \frac{1}{2}(s-\sqrt{\theta})=B$, and in like manner $\phi \frac{1}{2}\left(s^{\prime}+\sqrt{\theta}\right)=A^{\prime}, \phi \frac{1}{2}\left(s^{\prime}-\sqrt{\theta}\right)=B^{\prime}$, we have

$$
\begin{array}{ll}
F s=n(A+B)-\frac{s(A-B)}{\sqrt{\theta}}, & G s=\frac{A-B}{\sqrt{\theta}}, \\
F s^{\prime}=n\left(A^{\prime}+B^{\prime}\right)-\frac{s^{\prime}\left(A^{\prime}-B^{\prime}\right)}{\sqrt{\theta}}, & G s^{\prime}=\frac{A^{\prime}-B^{\prime}}{\sqrt{\theta}},
\end{array}
$$

and therefore

$$
F s G s^{\prime}-F s^{\prime} G s=n \frac{(A+B)\left(A^{\prime}-B^{\prime}\right)-(A-B)\left(A^{\prime}+B^{\prime}\right)}{\sqrt{\theta}}-\frac{\left(s-s^{\prime}\right)(A-B)\left(A^{\prime}-B^{\prime}\right)}{\theta} ;
$$

or reducing and dividing by $s-s^{\prime}$,

$$
-\frac{F s G s^{\prime}-F s^{\prime} G s}{s-s^{\prime}}=2 n \frac{A B^{\prime}-A^{\prime} B}{\sqrt{\theta}\left(s-s^{\prime}\right)}+\frac{(A-B)\left(A^{\prime}-B^{\prime}\right)}{\theta} .
$$


Hence, substituting for $A, B, A^{\prime}, B^{\prime}$ these values, we have the expression

$$
\begin{aligned}
2 n \frac{\phi \frac{1}{2}(s+\sqrt{\theta}) \phi \frac{1}{2}\left(s^{\prime}-\sqrt{\theta}\right)-\phi \frac{1}{2}(s-\sqrt{\theta}) \phi \frac{1}{2}\left(s^{\prime}+\sqrt{\theta}\right)}{\left(s-s^{\prime}\right) \sqrt{\theta}} \\
+\frac{\left\{\phi \frac{1}{2}(s+\sqrt{\theta})-\phi \frac{1}{2}(s-\sqrt{\theta})\right\}\left\{\phi \frac{1}{2}\left(s^{\prime}+\sqrt{\theta}\right)-\phi \frac{1}{2}\left(s^{\prime}-\sqrt{\theta}\right)\right\}}{\theta}
\end{aligned}
$$

which is of the form

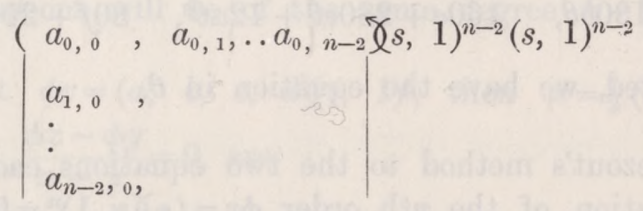

and equating to zero the determinant formed with the coefficients, we have an equation in $\theta$ which is the equation of differences of the given equation $\phi v=0$. For instance, if the given equation is $\phi v=(a, b, c, d \gamma v, 1)^{3}=0$, then we have

$$
\begin{gathered}
8 \phi \frac{1}{2}(s+\sqrt{\theta})=(a, 2 b+a \sqrt{\theta}, 4 c+4 b \sqrt{\theta}+a \theta, 8 d+12 c \sqrt{\theta}+6 b \theta+a \theta \sqrt{\theta} \gamma s, 1)^{3} \\
=(A, B, C, D \gamma s, 1)^{3}, \\
8 \phi \frac{1}{2}(s-\sqrt{\theta})=(a, 2 b-a \sqrt{\theta}, 4 c-4 b \sqrt{\theta}+a \theta, 8 d-12 c \sqrt{\theta}+6 b \theta-a \theta \sqrt{\theta} \gamma s, 1)^{3} \\
=\left(A^{\prime}, B^{\prime}, C^{\prime}, D^{\prime} \gamma s, 1\right)^{3} ;
\end{gathered}
$$

and the function in $s, s^{\prime}$ is

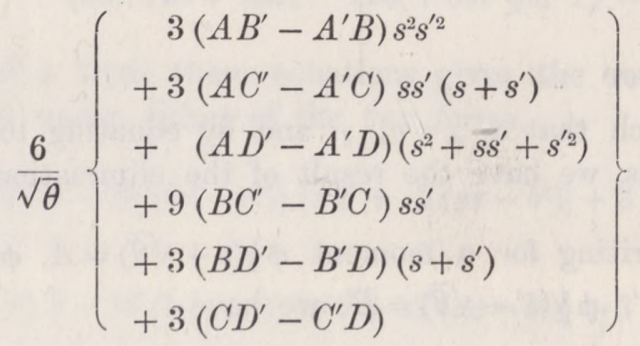

$$
\begin{aligned}
& +\frac{1}{\theta}\left(A-A^{\prime}, B-B^{\prime}, C-C^{\prime}, D-D^{\prime} \gamma s, 1\right)^{3}\left(A-A^{\prime}, B-B^{\prime}, C-C^{\prime}, D-D^{\prime} \gamma s^{\prime}, 1\right)^{3},
\end{aligned}
$$

which is equal to

$$
\begin{aligned}
& 12\left\{\begin{array}{l}
-3 a^{2} s^{2} s^{\prime 2} \\
-12 a b s s^{\prime}\left(s+s^{\prime}\right) \\
+\left(-12 a c-a^{2} \theta\right)\left(s^{2}+s s^{\prime}+s^{\prime 2}\right) \\
+\left(36 a c-72 b^{2}+9 a^{2} \theta\right) s s^{\prime} \\
+(24 a d-72 b c+12 a b \theta)\left(s+s^{\prime}\right) \\
+96 b d-144 c^{2}+\left(-48 a c+72 b^{2}\right) \theta-3 a^{2} \theta^{2}
\end{array}\right\} \\
& +4\left(3 a s^{2}+12 b s+12 c+a \theta\right)\left(3 a s^{\prime 2}+12 b s^{\prime}+12 c+a \theta\right) ;
\end{aligned}
$$


or reducing and dividing by 32 , this is

$$
\left\{9\left(a c-b^{2}\right)+3 a^{2} \theta\right\} s s^{\prime}+\{9(a d-b c)+6 a b \theta\}\left(s+s^{\prime}\right)+36\left(b d-c^{2}\right)+\left(-15 a c+27 b^{2}\right) \theta-a^{2} \theta^{2},
$$

the terms in $s^{2}, s^{\prime 2}$ disappearing, as they should do. Writing this under the form

$$
\left\{\begin{array}{ll}
9\left(a c-b^{2}\right)+3 a^{2} \theta, & 9(a d-b c)+6 a b \theta \\
9(a d-b c)+6 a b \theta, & 36\left(b d-c^{2}\right)+\left(-15 a c+27 b^{2}\right) \theta-a^{2} \theta^{2}
\end{array} \mid \zeta s, 1\right)\left(s^{\prime}, 1\right)
$$

and equating the determinant to zero, we have the required equation in $\theta$ : the form is that obtained by the ordinary process of applying Bezout's method to the two equations $\left.\left(3 a, 12 b, 12 c+a \theta^{\gamma}\right\} s, 1\right)^{2}=0,(3 b, 12 c+4 a \theta, 12 d+8 b \theta \gamma s, 1)^{2}=0$, being in fact the before-mentioned equation

$$
\left(a^{2} \theta^{2}+\left(15 a c-27 b^{2}\right) \theta-36\left(b d-c^{2}\right)\right)\left(a^{2} \theta+3\left(a c-b^{2}\right)\right)+3(2 a b \theta+3(a d-b c))^{2}=0 .
$$

But, as already remarked, this elimination process is less convenient for the complete

\begin{tabular}{|c|c|c|c|c|c|c|}
\hline$\theta^{10}$ & $\theta^{9} \cdot 100 \times$ & $\theta^{8} \cdot 50 \times$ & $\theta^{7} \cdot 2500 \times$ & $\theta^{6} \cdot 125 \times$ & $\theta^{5} \cdot 625 \times$ & $\theta^{4} \cdot \frac{1}{9} 2500 \times$ \\
\hline$A^{8}+1$ & $A^{6} C+1$ & $\begin{array}{l}A^{6} B+1 \\
A^{4} C^{2}+75\end{array}$ & $\begin{array}{l}A^{4} B C+1 \\
A^{2} F^{2}+1 \\
A^{2} C^{3}+29\end{array}$ & $\begin{array}{l}A^{4} H+16 \\
A^{4} B^{2}-19 \\
A^{2} B C^{2}+450 \\
C F^{2}+800 \\
C^{4}+6325\end{array}$ & $\begin{array}{l}A^{4} G+1 \\
A^{2} B^{2} C-160 \\
A F I+60 \\
A C^{2} D-1140 \\
B F^{2}+280 \\
B C^{3}+3620\end{array}$ & $\begin{array}{l}A^{3} J-212 \\
A^{2} B H-1545 \\
A^{2} B^{3}+230 \\
A E I+275 \\
B E F+1225 \\
B^{2} C^{2}+11650 \\
C^{2} H+1125\end{array}$ \\
\hline
\end{tabular}
development of the result, than the method first explained in the present memoir.

[The equation p. 257, changing the notation and inserting the omitted coefficients, becomes $0=$

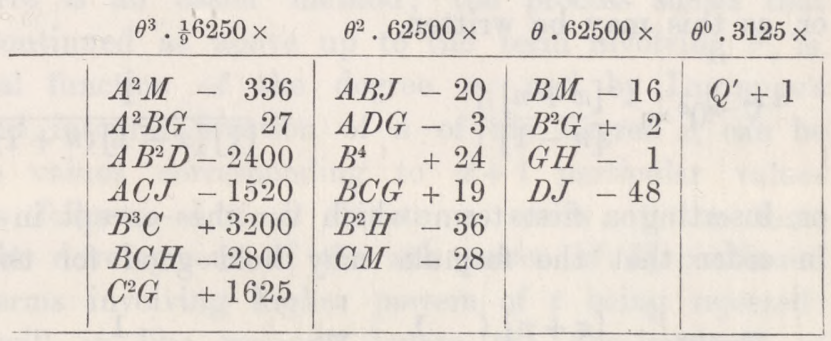

where the capital letters denote the covariants of the quantic as explained 141 and 143.] 